02

\title{
Дифференциальные характеристики спектров оптического пропускания кристаллов класса силленитов
}

\author{
(C) Т.А. Журин, Е.С. Сим, В.Г. Дю, М.Г. Кистенева, С.М. Шандаров \\ Томский государственный университет систем управления и радиоэлектроники, \\ 634050 Томск, Россия \\ e-mail: timoxazh1@gmail.com
}

Поступила в редакцию 15.03 .2020 г.

В окончательной редакции 15.05.2020 г.

Принята к публикации 20.05.2020 г.

\begin{abstract}
Представлены результаты сравнительного анализа дифференциальных характеристик измеренного экспериментально спектра оптического пропускания монокристаллического образца $\mathrm{Bi}_{12} \mathrm{GeO}_{20}$ с результатами расчета спектральных зависимостей фурье-компонент в его временном разложении при гармонической модуляции зондирующего излучения по длине волны.
\end{abstract}

Ключевые слова: германат висмута, оптическое пропускание, дифференциальные характеристики, численное моделирование.

DOI: $10.21883 /$ OS.2020.09.49861.91-20

\section{Введение}

Кристаллы класса силленитов $\mathrm{Bi}_{12} \mathrm{MO}_{20}(\mathrm{M}=\mathrm{Si}, \mathrm{Ge}$, Ti), обладающие фотопроводящими, фоторефрактивными и фотохромными свойствами, являются привлекательными материалами для исследования эффектов взаимодействия и самовоздействия световых пучков и реализации устройств фоторефрактивной нелинейной оптики и динамической голографии [1-16]. Эти фоточувствительные свойства связываются с дефектными центрами, энергетические уровни которых расположены в запрещенной зоне. Фотопроводимость в силленитах обеспечивается возбуждением с дефектов донорного типа электронов в зону проводимости, а их дрейф и захват на ловушечные центры приводит к фотоиндуцированному перераспределению зарядов, сопровождающемуся вследствие линейного электрооптического эффекта возмущениями показателя преломления, т.е. к фоторефрактивному эффекту $[1,4-7]$. Различия в сечениях фотоионизации доноров и ловушек, захвативших электроны, обусловливает фотохромные изменения спектра оптического поглощения, особенно заметные для кристаллов $\mathrm{Bi}_{12} \mathrm{TiO}_{20}$ и $\mathrm{Bi}_{12} \mathrm{SiO}_{20}[2,3,7,17]$. В этих кристаллах обнаружен также вклад в оптическое поглощение, зависящий от внешних воздействий, таких как температурный отжиг и оптическая засветка, и связанный с внутрицентровыми переходами $[17,18]$. Описание наблюдаемых спектральных зависимостей оптического поглощения в монокристаллических образцах силленитов $\mathrm{Bi}_{12} \mathrm{GeO}_{20}, \mathrm{Bi}_{12} \mathrm{SiO}_{20}$ и $\mathrm{Bi}_{12} \mathrm{TiO}_{20}$ : $\mathrm{Al}$, принимающее во внимание вклады в него как процессов фотовозбуждения электронов в зону проводимости с донорных центров, так и внутрицентровых переходов [17], показало близость параметров последних для этих кристаллов. Предположительно, такие внутрицентровые переходы имеют общую природу и происходят между уровнями структурных дефектов, связанными с ионами висмута, к которым, как к возможным центрам люминесценции в стеклах и монокристаллах, проявляется значительный интерес [19].

Определение типов дефектных центров и измерение их параметров в силленитах прямыми методами затруднено тем, что оптические спектры этих кристаллов характеризуются широкими перекрывающимися полосами поглощения. Идентификация дефектных центров, вносящих вклад в примесное оптическое поглощение, возможна при использовании метода модуляционной (дифференциальной) спектроскопии [20,21], основанного на анализе временного спектра отклика среды на малые гармонические возмущения длины волны входного сигнала ( $\lambda$-модуляция). Экспериментальные исследования методом $\lambda$-модуляционной спектроскопии образцов $\mathrm{Bi}_{12} \mathrm{SiO}_{20}: \mathrm{Fe}$ позволили, например, определить положение примесных уровней в запрещенной зоне данного кристалла [22]. Проведенное в [23] численное моделирование временных зависимостей коэффициента пропускания образца $\mathrm{Bi}_{12} \mathrm{SiO}_{20}$ при гармонической модуляции возбуждающего излучения по длине волны (с использованием модели оптического поглощения [17]) продемонстрировало возможность идентификации методом $\lambda$-модуляции внутрицентровых переходов в диапазоне $640-1000 \mathrm{~nm}$, где вклад в поглощение фотовозбуждения электронов с донорных центров относительно невелик.

Целью настоящей работы является сравнительный анализ дифференциальных характеристик полученного экспериментально спектра оптического пропускания монокристаллического образца $\mathrm{Bi}_{12} \mathrm{GeO}_{20}$ с результатами расчета спектральных зависимостей фурье-компонент в его временном разложении при гармонической модуля- 
ции зондирующего излучения по длине волны, проведенного в рамках модели примесного поглощения [17].

\section{Методика эксперимента и численного моделирования}

В экспериментах по исследованию спектральной зависимости коэффициента пропускания $T_{e}(\lambda)$ использовался плоскопараллельный монокристаллический образец $\mathrm{Bi}_{12} \mathrm{GeO}_{20}$ (BGO) с толщиной $d=10.5 \mathrm{~mm}$, который имел оптически полированные грани с ориентацией (100). Измерения проводились с помощью спектрофотометра СФ-56 в диапазоне 570-950 nm при комнатной температуре. Полученный спектр пропускания $T_{e}(\lambda)$ исследованного образца BGO представлен точками на рис. 1 (зависимость 1). Исходные экспериментальные данные для $T_{e}(\lambda)$ сглаживались с помощью встроенной в программу MathCad функции ksmooth и далее подвергались дифференцированию, результаты которого для $d T_{e} / d \lambda$, показаны штриховой кривой 1 на рис. 2. Вторая производная коэффициента пропускания $d^{2} T_{e} / d \lambda^{2}$, представленная кривой 2 на рис. 1, имеет характерные максимумы на длинах волн $\lambda=619,701,755$ и $835 \mathrm{~nm}$.

В случае гармонического закона $\lambda$-модуляции зондирующего излучения с частотой $f$ амплитуда второй гармоники $T_{m}^{(2 f)}\left(\lambda_{0}\right)$ временного спектра в прошедшем образец световом пучке на частоте $2 f$ пропорциональна второй производной коэффициента пропускания $d^{2} T / d \lambda^{2}$. Поэтому для определения спектрального положения полос поглощения, соответствующих внутрицентровым переходам в кристалле $\mathrm{Bi}_{12} \mathrm{SiO}_{20}$, в работе [23] использовался расчет зависимости амплитуды второй гармоники $T_{m}^{(2 f)}\left(\lambda_{0}\right)$ от центральной длины волны зондирующего пучка $\lambda_{0}$. Реализация данной методики для исследуемого образца BGO осуществлялась аппроксимацией спектра его показателя поглощения $k(\lambda)$,

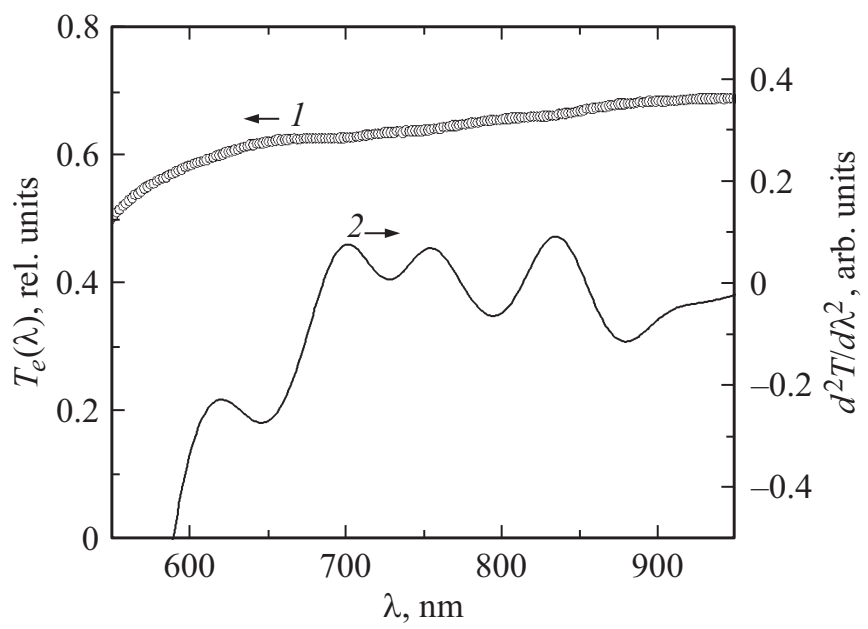

Рис. 1. Экспериментальная спектральная зависимость коэффициента пропускания $T_{e}(\lambda)(1)$ и спектральная зависимость его второй производной $d^{2} T_{e} / d \lambda^{2}(2)$ для кристалла $\mathrm{Bi}_{12} \mathrm{GeO}_{20}$.

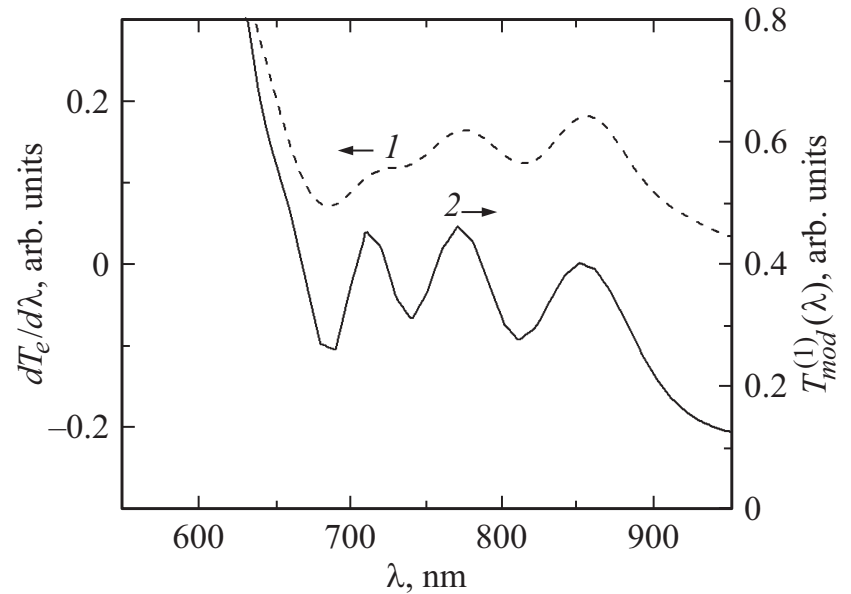

Рис. 2. Спектральные зависимости первой производной $d T_{e} / d \lambda(1)$ и амплитуды первой фурье-гармоники $T_{\bmod }^{(1)}\left(\lambda_{0}\right)(2)$ оптического пропускания для кристалла $\mathrm{Bi}_{12} \mathrm{GeO}_{20}$ в режиме гармонической $\lambda$-модуляции зондирующего пучка с амплитудой $\Delta \lambda=10 \mathrm{~nm}$.

рассчитываемого из экспериментальных значений для коэффициента пропускания $T_{e}(\lambda)$ (зависимость 1 на рис. 1), с использованием подхода, описанного в [17]. Процесс аппроксимации потребовал учета четырех внутрицентровых переходов с гауссовой формой частотной зависимости и с максимумами на длинах волн $\lambda_{m}^{i c}=645$, 700,754 и $827 \mathrm{~nm}$. Далее из спектра $k(\lambda)$, являющегося непрерывной функцией, вычислялась временная зависимость коэффициента пропускания плоскопараллельного образца для случая гармонической $\lambda$-модуляции зондирующего пучка с амплитудой $\Delta \lambda$ и центральной длиной волны $\lambda_{0}[23]$ :

$$
T_{\text {mod }}\left(\lambda_{0}, t\right)=\frac{\left[1-R\left(\lambda_{0}, t\right)\right]^{2} \exp \left[-k\left(\lambda_{0}, t\right) d\right]}{1-R^{2}\left(\lambda_{0}, t\right) \exp \left[-2 k\left(\lambda_{0}, t\right) d\right]},
$$

где $R\left(\lambda_{0}, t\right)$ - френелевский коэффициент отражения от граней образца по интенсивности при нормальном падении светового пучка.

Временные зависимости $T_{\bmod }\left(\lambda_{0}, t\right)$ рассчитывались для $\lambda_{0}$, изменяющейся в спектральном диапазоне от 570 до $950 \mathrm{~nm}$, при значении амплитуды модуляции $\Delta \lambda=10 \mathrm{~nm}$. Далее они аппроксимировались разложением в ряд Фурье, содержащим постоянную составляющую $T_{\text {mod }}^{(0)}\left(\lambda_{0}\right)$ и четыре гармоники частоты модуляции с частотами $n f(n=1,2,3,4)$ и соответствующими амплитудами $T_{\text {mod }}^{(n)}\left(\lambda_{0}\right)$, с использованием метода наименьших квадратов. Спектральные зависимости для амплитуд $T_{\text {mod }}^{(0)}\left(\lambda_{0}\right)$ и $T_{\text {mod }}^{(2)}\left(\lambda_{0}\right)$ представлены на рис. 3 кривыми 1 и 2 соответственно, а зависимость $T_{\bmod }^{(1)}\left(\lambda_{0}\right)$ показана на рис. 2 сплошной кривой 2. Характерно, что на кривой 2 на рис. 3 максимумы для амплитуды второй гармоники имеют место при $\lambda_{0}=594,653,699,754$ и $829 \mathrm{~nm}$. 


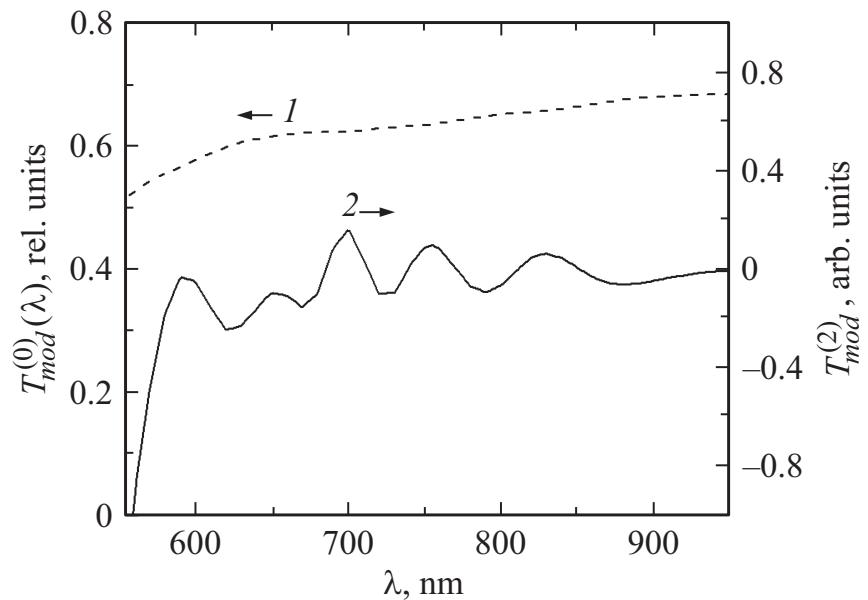

Pис. 3. Спектральные зависимости для амплитуд фурьегармоник оптического пропускания кристалла $\mathrm{Bi}_{12} \mathrm{GeO}_{20}$ $T_{\text {mod }}^{(0)}\left(\lambda_{0}\right)(1)$ и $T_{\text {mod }}^{(2)}\left(\lambda_{0}\right)(2)$ в режиме гармонической $\lambda$-модуляции зондирующего пучка с амплитудой $\Delta \lambda=10 \mathrm{~nm}$.

\section{Обсуждение результатов}

Экспериментальная зависимость 1 для коэффициента пропускания $T_{e}(\lambda)$ исследуемого образца BGO на рис. 1 является достаточно гладкой, а присутствующие на ней плавные отклонения от монотонного поведения не позволяют идентифицировать структурные особенности, обусловленные вкладами центров, различающихся по типу и спектральному положению максимумов поглощения. Однако на спектральной зависимости первой производной коэффициента пропускания $d T_{e} / d \lambda$ (кривая 1 на рис. 2) отклонения от монотонности являются хорошо различимыми. Анализ этой зависимости показывает, что наблюдаемые на ней экстремумы не удается сопоставить со спектральными максимумами, характеризующими используемые в численной аппроксимации энергетические параметры как доноров, так и дефектов, описывающих внутрицентровые переходы. Такое сопоставление для спектральной зависимости второй производной коэффициента пропускания $d^{2} T_{e} / d \lambda^{2}$ (кривая 2 на рис. 1) показывает, что положение трех наблюдаемых на ней максимумов (при $\lambda=701,755$ и $835 \mathrm{~nm}$ ) близко к максимумам гауссовых компонент, полученных при численной аппроксимации показателя поглощения $k(\lambda)$ и определяющих вклад в него внутрицентровых переходов с $\lambda_{m}^{i c}=700,754$ и $827 \mathrm{~nm}$. Максимум при $\lambda=619 \mathrm{~nm}$ на кривой 2 на данном рис. 1 заметно отличается от полученного при аппроксимации значения $\lambda_{m}^{i c}=645 \mathrm{~nm}$, что связано в первую очередь со значительным вкладом в примесное поглощение в спектральной области 570-650 nm процессов фотовозбуждения электронов в зону проводимости с донорных центров, а также с его сильной зависимостью от длины волны.

При численном моделировании временного спектра в прошедшем образец BGO световом пучке, наблюда- емом при гармонической $\lambda$-модуляции зондирующего излучения, зависимость для нулевой гармоники $T_{\bmod }^{(0)}\left(\lambda_{0}\right)$ (кривая 1 на рис. 3) с достаточной точностью совпадает со спектром коэффициента пропускания исследуемого образца $T_{e}(\lambda)$ (зависимость 1 на рис. 1 ). Можно также отметить, что максимумы на спектральной зависимости для первой временной гармоники $T_{\bmod }^{(1)}\left(\lambda_{0}\right)$ (кривая 2 на рис. 2) являются близкими по положению к таковым для спектра $d T_{e} / d \lambda$, представленного на том же рис. 2 кривой 1 , но более ярко выраженными.

Сравнение спектральных зависимостей для второй производной $d^{2} T_{e}(\lambda) / d \lambda^{2}$ (кривая 2 на рис. 1) и для второй временной гармоники $T_{\bmod }^{(2)}\left(\lambda_{0}\right)$ (кривая 2 на рис. 3) показывает, что в области 680-950 nm они качественно согласуются друг с другом. Спектральное положение трех максимумов для амплитуды второй гармоники $T_{\mathrm{mod}}^{(2)}\left(\lambda_{0}\right)$ в этой области (с $\lambda_{0}=699,754$ и $829 \mathrm{~nm})$ отличается от использованных значений $\lambda_{m}^{i c}$ при численной аппроксимации показателя поглощения $k(\lambda)$ исследуемого кристалла BGO не более чем на $2 \mathrm{~nm}$.

\section{Заключение}

Таким образом, в работе проведен анализ дифференциальных характеристик экспериментально измеренного спектра оптического пропускания монокристаллического образца $\mathrm{Bi}_{12} \mathrm{GeO}_{20}$ с толщиной $d=10.5 \mathrm{~mm}$. Получено, что вторая производная коэффициента пропускания исследованного образца имеет четыре максимума, три из которых близки по спектральному положению к максимумам гауссовых компонент, найденных при численной аппроксимации его показателя поглощения и определяющих вклад в последний внутрицентровый переход. Рассмотренный подход позволяет идентифицировать внутрицентровые переходы между уровнями возможных центров люминесценции и в других кристаллах класса силленитов.

Для оценки возможности экспериментальной идентификации внутрицентровых переходов методом $\lambda$-модуляции в работе проведено численное моделирование временных зависимостей коэффициента пропускания исследованного образца $\mathrm{Bi}_{12} \mathrm{GeO}_{20}$ при гармонической модуляции зондирующего излучения по длине волны с использованием модели оптического поглощения силленитов, рассмотренной в [17]. Проведенный сравнительный анализ показал, что оба подхода дают близкие значения для спектрального положения максимумов гауссовых компонент, описывающих внутрицентровые переходы в $\mathrm{Bi}_{12} \mathrm{GeO}_{20}$ в диапазоне 640-1000 nm, где вклад в оптическое поглощение процессов фотовозбуждения электронов с донорных центров относительно невелик.

\section{Финансирование работы}

Работа выполнена при финансовой поддержке Министерства науки и высшего образования Российской 
Федерации в рамках Госзадания на 2020-2022 годы (задание FEWM-2020-0038/3).

\section{Конфликт интересов}

Авторы заявляют, что у них нет конфликта интересов.

\section{Список литературы}

[1] Петров М.П., Степанов С.И., Хоменко А.В. Фоторефрактивные кристаллы в когерентной оптике. СПб.: Наука, 1992. $320 \mathrm{c}$

[2] Малиновский В.К., Гудаев О.А., Гусев В.А., Деменко С.И. Фотоиндуцированные явления в силленитах. Новосибирск: Наука, 1990. 160 с.

[3] Каргин Ю.Ф., Бурков В.И., Марьин А.А., Егорышева А.В. Кристаллы $\mathrm{Bi}_{12} \mathrm{M}_{x} \mathrm{O}_{20+\delta}$ со структурой силленита. Синтез, строение, свойства. М.: Изд-во ИОНХ РАН, 2004. 316 с.

[4] Stepanov S.I. // Repts. Progr. Phys. 1994. V. 57. P. 39. doi org/10.1088/0034-4885/57/1/002

[5] Solymar L., Webb D.J., Grunnet-Jepsen A. The Physics and Application of Photorefractive Materials. Oxford: Clarendon Press, 1996. $512 \mathrm{p}$.

[6] Petrov M.P., Bryksin V.V. // Photorefractive Materials and Their Applications 2. / Ed. by Gunter P., Huignard J.P. Berlin: Springer-Verlag, 2007. P. 285

[7] Шандаров С.М., Шандаров В.М., Мандель А.Е., Буримов Н.И. Фоторефрактивные эффекты в электрооптических кристаллах. Томск: ТУСУР, 2007. 242 с.

[8] Шандаров С.М., Буримов Н.И., Кульчин Ю.Н., Ромаико Р.В., Толстик А.Л., Шепелевич В.В. // Квант. электрон. 2008. № 38. C. 1059.

[9] Колегов А.А., Шандаров С.М., Симонова Г.В., Кабанова Л.А., Буримов Н.И., Шмаков С.С., Быков В.И., Каргин Ю.Ф. // Квант. электрон. 2011. № 41. С. 847.

[10] Брюшинин М.А., Куликов В.В., Соколов И.A., Delaye P., Pauliat G. // ФТT. 2014. № 56. C. 1158; Bryushinin M.A., Kulikov V.V., Sokolov I.A., Delaye P., Pauliat G. // Phys. Solid State. 2014. V. 56. N 6. P. 1206. doi $10.1134 / \mathrm{S} 1063783414060092$

[11] Kamshilin A.A., Raita E., Prokofiev V.V., Jaaskelainen T. // Appl. Phys. Lett. 1995. N 67. P. 3242. doi 10.1063/1.114885

[12] Iturube-Castillo M.D., Marquez-Aguilar P.A., SanchezMondragon J.J., Stepanov S.I., Vysloukh V.A. // Appl. Phys. Lett. 1994. N 64. P. 408. doi org/10.1063/1.111163

[13] Шепелевич В.В., Коваршик Р., Кислинг А., Матусевич В., Голуб А.А. // Квант. электрон. 2003. N 33 С. 446; Shepelevich V.V. et al. // Quantum Electronics. 2003. N 33(5). P. 446. doi org/10.1070/QE2003v033n05ABEH002432

[14] Fazio E., Ramadan W., Belardini A., Bosco A., Bertolotti M., Petris A., Vlad V. // Phys. Rev. E. 2003. N 67. 026611. doi org/10.1103/PhysRevE.67.026611

[15] Garcia-Quirino G.C., Iturube-Castillo M.D., Vysloukh V.A., Sanchez-Mondragon J.J., Stepanov S.I., Lugo-Martinez G., Torres-Cisneros G.E. // Opt. Lett. 1997. N 22. P. 154. doi org/10.1364/OL.22.000154
[16] Marinova V., Huei Lin S., Chung Liu R., Hsu K.Y. // Photorefractive Effect: Principles, Materials, and NearInfrared Holography. John Wiley \& Sons, Inc. 2016. P. 1. doi 10.1002/047134608X.W6010.pub2

[17] Кистенева М.Г., Худякова Е.С., Шандаров С.М., Акрестина А.С., Дю В.Г., Каргин Ю.Ф. // Квант. электрон. 2015. T. 45. № 7. C. 685; Kisteneva M.G., Khudyakova E.S., Shandarov S.M., Akrestina A.S., Duy V.G., Kargin Yu.F. // Quantum Electronics. 2015. T. 45. N 7. C. 685. doi 10.1070/QE2015v045n07ABEH015521

[18] Kisteneva M.G., Akrestina A.S., Shandarov S.M., Smirnov S.V., Bikeev O.N., Lovetskii K.P., Kargin Yu.F. // J. Holography and Speckle. 2009. N 5. P. 280. doi 10.1166/jhs.2009.1029

[19] Дианов Е.М. // Квант. электрон. 2010. Т. 40. № 4. С. 283; Dianov E.M. // Quantum Electronics. 2010. V. 40. N 4. P. 283. doi org/10.1070/QE2010v040n04ABEH014304

[20] Кардона М. Модуляционная спектроскопия. М.: Мир, 1972. $414 \mathrm{c}$.

[21] Георгобиани А.Н. // Соросовский образовательный журнал. 2001. № 6. С. 75.

[22] Petkova P., Kostova B., Marinova V., Tacheva J. // IOP Conf. Ser:: Mater. Sci. Eng. 2010. V. 15. P. 012070. doi 10.1088/1757-899X/15/1/012070

[23] Сим Е.С., Кистенева М.Г., Журин Т.А., Шандаров С.М. // Изв. вузов. Физика. 2019. Т. 62. № 2. C. 117; $\operatorname{Sim}$ E.S., Kisteneva M.G., Zhurin T.A., Shandarov S.M. // Russ. Phys. J. 2019. V. 62. N 2. P. 132. doi org/10.1007/s11182-019-01693-0 